\title{
The Epidemiology of Herpes Zoster
}

\author{
M. COOPER
}

London

\begin{abstract}
Summary
The epidemiology of Zoster is analysed by means of data acquired from the Zoster Clinic at Moorfields and the RCGP Research Unit. It complements and extends previous surveys, using much larger and consistent data, showing the effects of age, sex and season. The behaviour in the population as a whole and the pathogenesis are discussed; a model is proposed to explain the characteristics of some of the data, particularly the effects of age and non-specific stresses.
\end{abstract}

In the literature there is confusion and little information about the epidemiology of Herpes Zoster (HZ). This investigation was encouraged by hearing that one of our Zoster Clinic patients had been awarded an industrial injury settlement on the grounds that her affliction had been caused by her exposure to chickenpox (she was a teacher). A recent report on a Zoster cluster, complete with correspondence for and against, has done little to

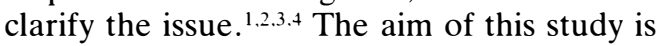
firstly to look at the epidemiological behaviour of $\mathrm{HZ}$ and then to suggest a new model which may account for some of the observations, particularly those related to age.

It is accepted that $\mathrm{HZ}$ is not usually caught by contact with chickenpox and does not follow its characteristic epidemic pattern. It does not have any sex preference but is commoner with increasing age. ${ }^{5.6 .7}$ The definitive paper by Hope-Simpson ${ }^{5}$ describes his experiences of 162 cases seen in general practice over a period of 15 years. HZ incidence rose from $0.74 / 1,000$ under 10 years to a plateau of 2.5 between 20 and 50 and then rose again to 10 by 80 ; the reasons for this behaviour are discussed. For a life span of 85 years, there was a 50 per cent probability of acquiring $\mathrm{HZ}$ once and a 1 per cent chance of getting it twice (4 per cent of cases were second attacks). The model of pathogenesis he proposed, that of viral reactivation due to loss of immunity, has not been improved upon to date.

Since there are no comparable recent data, we present the experience from the Zoster Clinic at Moorfields Eye Hospital, London and from the Weekly Returns of Infectious Diseases monitored by the Royal College of General Practitioners Research Unit. ${ }^{8}$

\section{Material and Methods}

The Zoster Clinic has been functioning for the last 15 years and during this time consistent records have been kept for nearly all attendances. These were recently put into computer storage, which has made overall statistical assessment and recall much easier. To date, about 1,200 patient records have been entered.

The Weekly Returns Service of the RCGP provides data from 1967 and now covers a population of over 200,000 in 39 general practices scattered around England (33), Wales (4), Scotland (1) and $\mathrm{N}$. Ireland (1). The figures for the last 3 years are accessible from computer, giving weekly numbers broken down by sex and 5 age groups. $\mathrm{HZ}$ and chickenpox are fortunately included in these returns.

The data were prepared in graphical form for the sake of clarity, and no statistical tests were performed. 


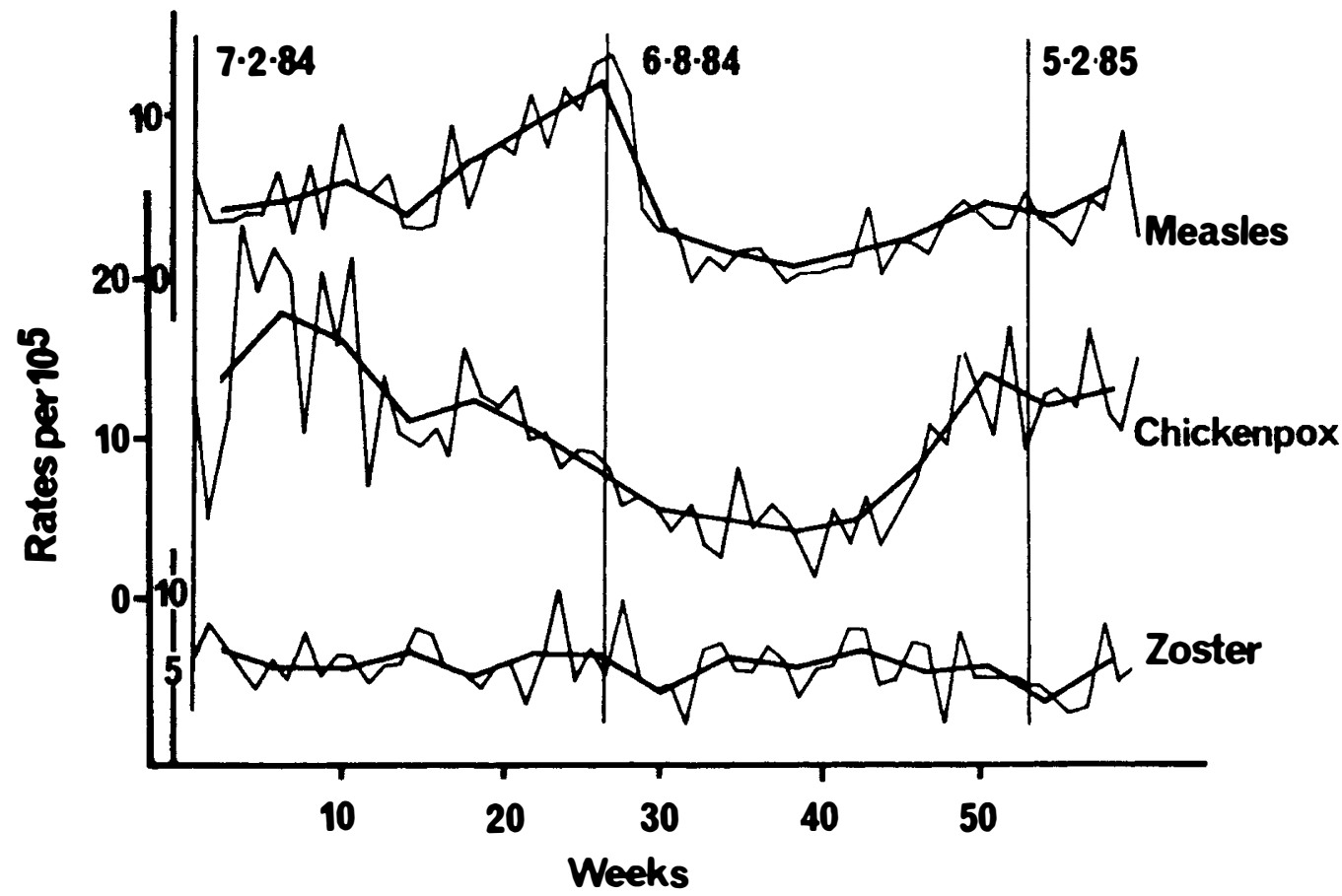

Fig. 1. Incidence rates for the general population of measles, chickenpox and zoster over a one-year period at weekly and monthly intervals.

\section{Results}

Figure 1 compares the weekly and monthly incidences of $\mathrm{HZ}$, chickenpox and measles over a year. ${ }^{9}$ There are obvious and different peaks for the latter two, but no apparent change corresponding to these in the $\mathrm{HZ}$ line.

Figure 2 compares the incidences of $\mathrm{HZ}$ and chickenpox in 1983 for both sexes. ${ }^{8}$ The general pattern is similar to Fig. 1 for both sexes, although $\mathrm{HZ}$ is slightly commoner in females, and chickenpox in males. This is similar to other years (not shown).

Figure 3 shows the age-group incidence for HZ and chickenpox over 1983. ${ }^{8}$ Not surprisingly, epidemic chickenpox is commonest in the under 14 year group and $\mathrm{HZ}$ in the over 45 year one. The 15-44 year age group has the lowest incidence for both and does not seem to follow the epidemic pattern at all, even for chickenpox.

Figure 4 compares the hours of sunshine recorded at Richmond over a year with the incidence of $\mathrm{HZ}$ over the same period. ${ }^{10}$ There is no obvious correlation.

Figure 5 depicts figures from the Zoster Clinic: the lower one the attendances per month over a year and the upper, the average disease 'score' (the sum of clinical involvement translated into a number by use of a standard scoring system) for each month over the same period. Attendances seem to be well spread throughout the year, but significant disease is slightly more common at the turn of the year, which is in accordance with clinical impression. The 'dip' in spring corresponds with the peak in chickenpox incidence.

Figure 6 collates the yearly rates for $\mathrm{HZ}$ and chickenpox between 1967 and 1983. ${ }^{11}$ The former seems to stay constant, whereas the latter has periodic peaks, but the rates are of the same order of magnitude when considered over the whole period.

Figure 7 shows the treatment of Zoster Clinic attendance figures compiled for the last 15 years. In view of the relatively constant rate for $\mathrm{HZ}$, it was felt justified to put all these together and form an age-number curve for both sexes (a). In order to convert these to rates per age-group, it was assumed that the population structure was similar to that of Greater London. ${ }^{12}$ The vertical scale, obviously, is not a true representation of inci- 


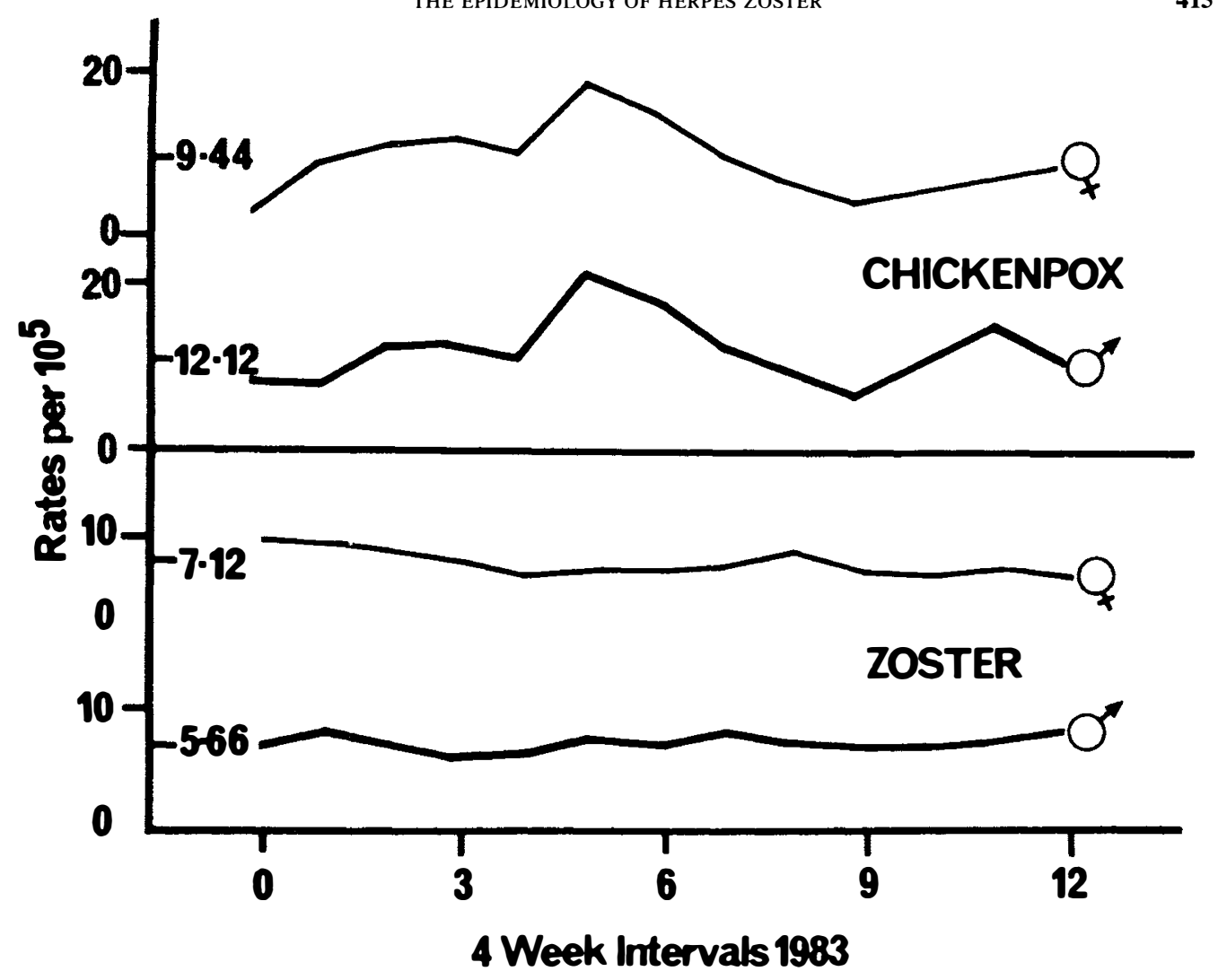

Fig. 2. Incidence rates by sex over 1983 for chickenpox and zoster (monthly).

dence rates, but does allow internal comparisons (b). The last graph (c) is (b) with a log vertical scale. Despite the assumptions, it seems reasonable to infer an exponential increase in $\mathrm{HZ}$ rate up to about 60 years of age, then a plateau.

Figure 8 shows the same treatment as in Figure 7 for some Mortality Returns to the Registrar General in 1981.13 These are for males; those for females are much the same, surprisingly. The true rates have been altered by shifting the curves up or down so that they coincide at the 45-49 year age group. This does not alter the shape of the curves, but makes comparison with each other and the family of cumulative normal distributions easier.

\section{Discussion}

\section{Epidemiology}

These data do not support horizontal transmission of Varicella-Zoster Virus (VSV) as the cause of $\mathrm{HZ}$, even with a lapse of 10 years, but they cannot refute it for the small percentage of cases ${ }^{5}$ which the recent report and correspondence in the Lancet ${ }^{1.2 .3 .4}$ have concentrated upon. Here, the main arguments concern the likelihood of clusters occurring by chance, but the conclusions depend upon which population is studied. In fact, it is possible to put forward a model which allows for clustering but does not implicate VSV directly.

The data do not support a seasonal pattern of occurrence either. The idea may have been engendered by $\mathrm{HZ}$ being seasonally more severe, hence increasing the clinical workload at particular times (in this case, around the New Year). The data are from Zoster Clinic attendances and represent a selected population; if anything, one might expect the number of referrals at this time of year to decrease because a lot of the sufferers are elderly and try to avoid travelling in the winter. The fact that numbers are maintained 


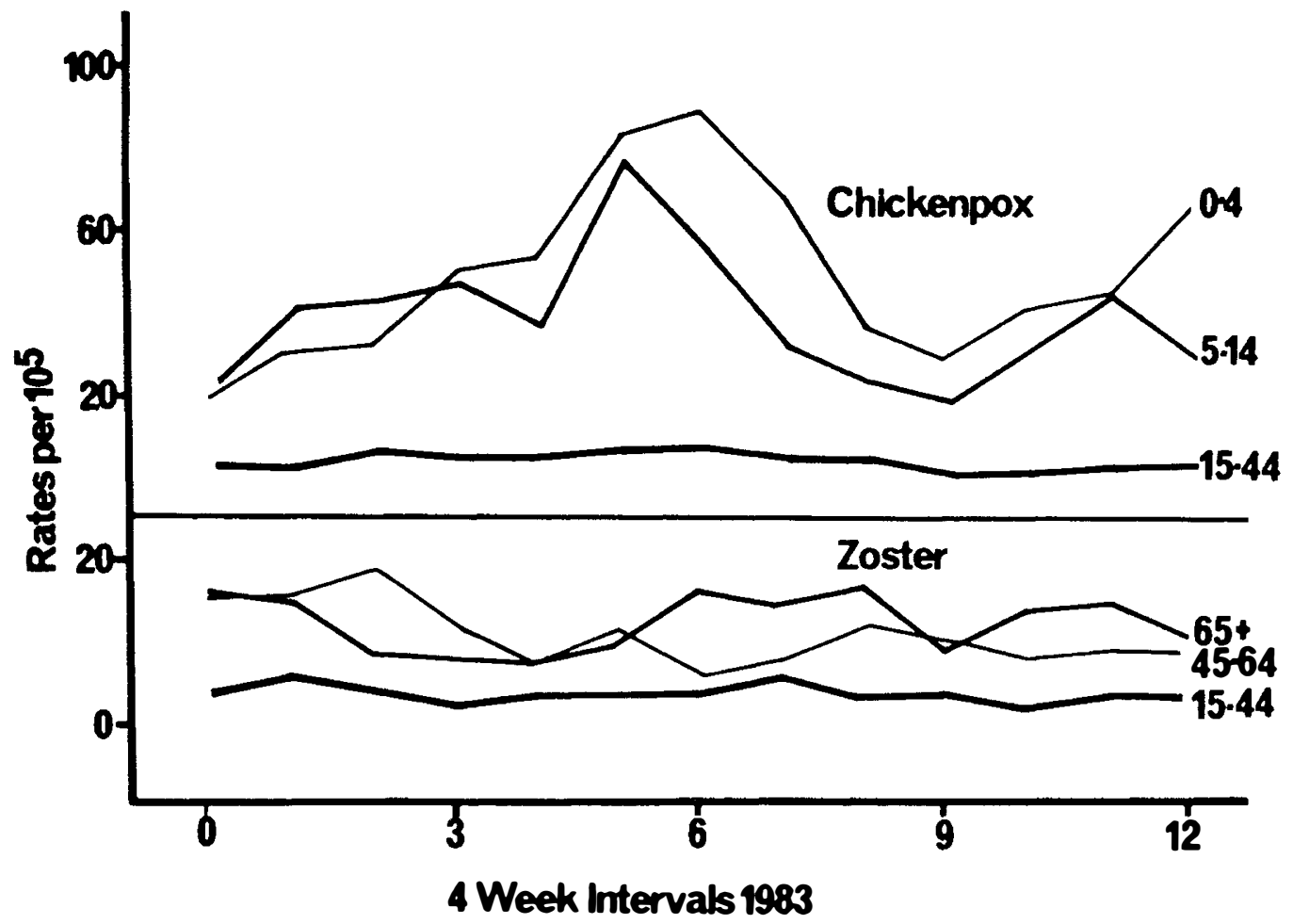

Fig. 3. Incidence rates by age over 1983 for chickenpox and zoster (monthly).

could mean that there is indeed a peak at this time. In support of our data, those that do come might be expected to have more severe disease either because they have not had early treatment or only they have sufficient reason to overcome the aversion to travel. The figures from the RCGP do not suggest any variation in $\mathrm{HZ}$ incidence throughout the year, but they may also be open to some bias.

There is a small difference in incidence between males and females and it is interesting that the bias is opposite for $\mathrm{HZ}$ and chickenpox; there seems to be no good reason for this.

The lack of correlation with hours of sunshine makes it unlikely that UV light has any role in Zoster, unlike findings in Herpes Simplex.

The age-incidence figures for chickenpox and $\mathrm{HZ}$ display the different epidemiological behaviours well. The former has a characteristic epidemic pattern affecting the young and the latter a relatively constant rate affecting the elderly. The 15-44 year age group is least affected, but those with chickenpox do not appear to follow the pattern of the younger groups. This may be simply because the scale and statistical variation hide any small peak or it may reflect non-infectious factors as the main disease determinants. It could be that these 'late' childhood fevers are second episodes of chickenpox, occurring because of loss of immunity, or may even be episodes of atypical disseminated $\mathrm{HZ}$. There is no way of deciding between these or any other possibilities from the available figures.

Looking at the population as a whole, the average yearly rate per 1,000 over the last 17 years is about 5 for chickenpox and that for $\mathrm{HZ}$ is 70 per cent of this. Over a lifetime of 80 years, about 40 per cent will acquire chickenpox and 30 per cent $\mathrm{HZ}$ at some stage. This ignores second and third attacks, which only make up 4 per cent of attendances and so have little influence. Ophthalmic involvement occurs in 15-20 per cent, so about 5 per cent of the population will acquire it at some stage in their life. In urban areas at least, ${ }^{6}$ there is serological evidence of contact with VZV in 95 per cent, so under-reporting is likely or at 


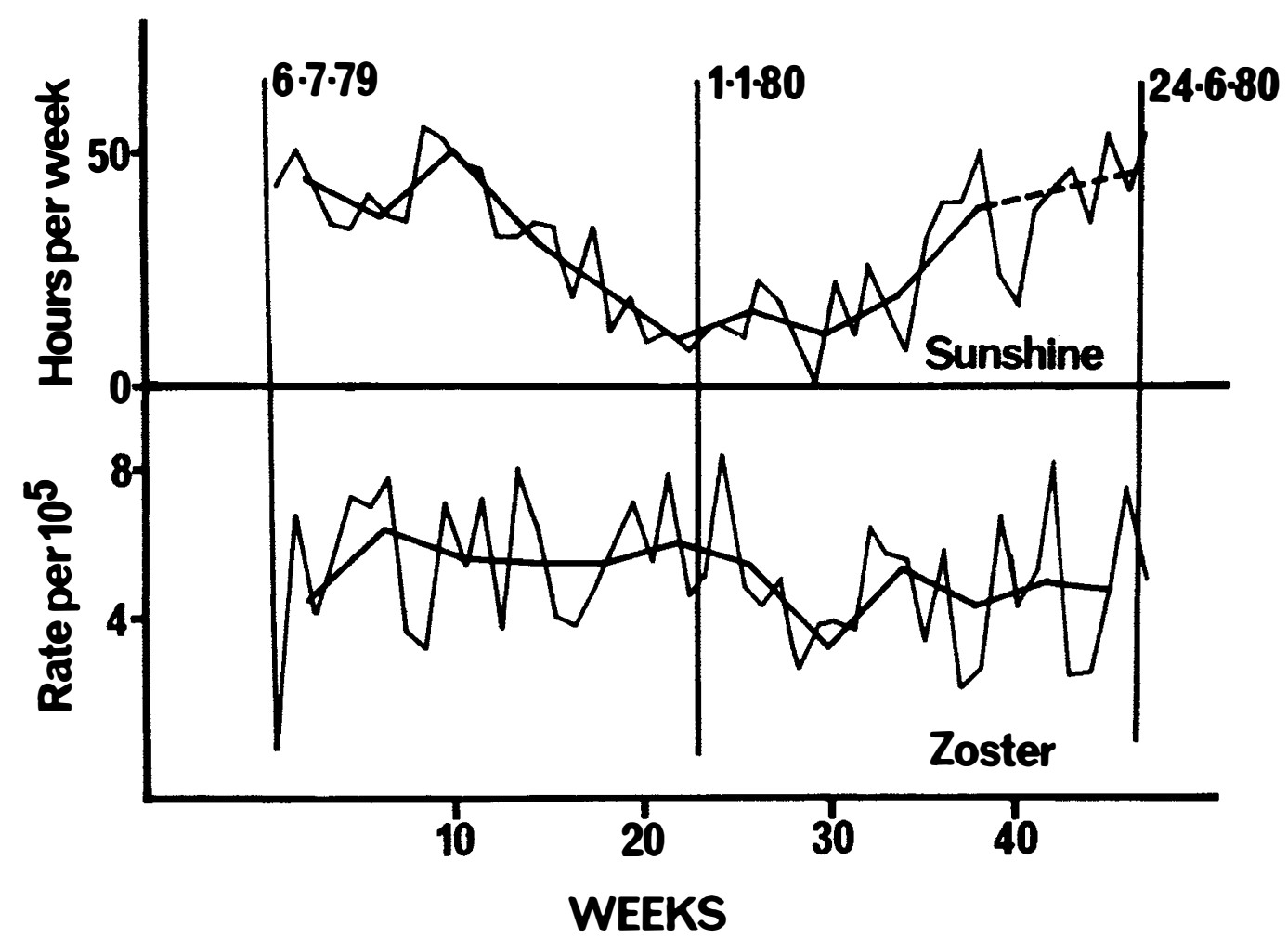

Fig. 4. Incidence rates of zoster and hours of sunshine over the same period of a year, weekly and monthly.

least half of chickenpox infection is sub-clinical. If anything, our figures are likely to underestimate the true rates.

\section{Pathogenesis}

During the initial infection with VSV, the virus is thought to ascend the sensory nerves and establish itself in the ganglia, either as chronic or latent infection. The purely sensory involvement and the tendency for the $\mathrm{HZ}$ rash to occur more commonly in the regions where the chickenpox rash is most dense support this. Whether sensory nerves other than cutaneous can be involved in this way (or why they are not) has not been discussed. It may be that some pain syndromes arise from occult $\mathrm{HZ}$ affecting non-cutaneous nerves. Motor nerves appear to be affected in a different way, perhaps as an epiphenomenon.

Assuming that most of our urban population come into contact with VZV and that just under half have clinical chickenpox, a large proportion will have established some sort of colonisation in their sensory ganglia, probably several. What determines when the VZV will reactivate and lead to clinical $\mathrm{HZ}$ ? There are many things that have been associated with $\mathrm{HZ}$, but there is usually nothing specific and certainly nothing consistent. The current idea of viral activation is based on alteration of immunity; the virus 'reverts' to an active form from time to time but the host immune system is capable of containing it and so prevents any clinical disease. When the system 'runs down' too far to do this, overt damage occurs and $\mathrm{HZ}$ results. There is still an anamnesic response, which may account for the clinical differences of $\mathrm{HZ}$ and chickenpox.

Stresses and non-specific trauma may affect the immune system and can be incorporated into a model of pathogenesis. The curves with a 'log' vertical scale in Figures 7 and 8 are known as Gompertz plots and have been used in analysing models of mortality. The exponential relation is best modelled by the Maxwell-Boltzman equation for energy distribution in gases, where the proportion of molecules above a certain energy (equivalent 


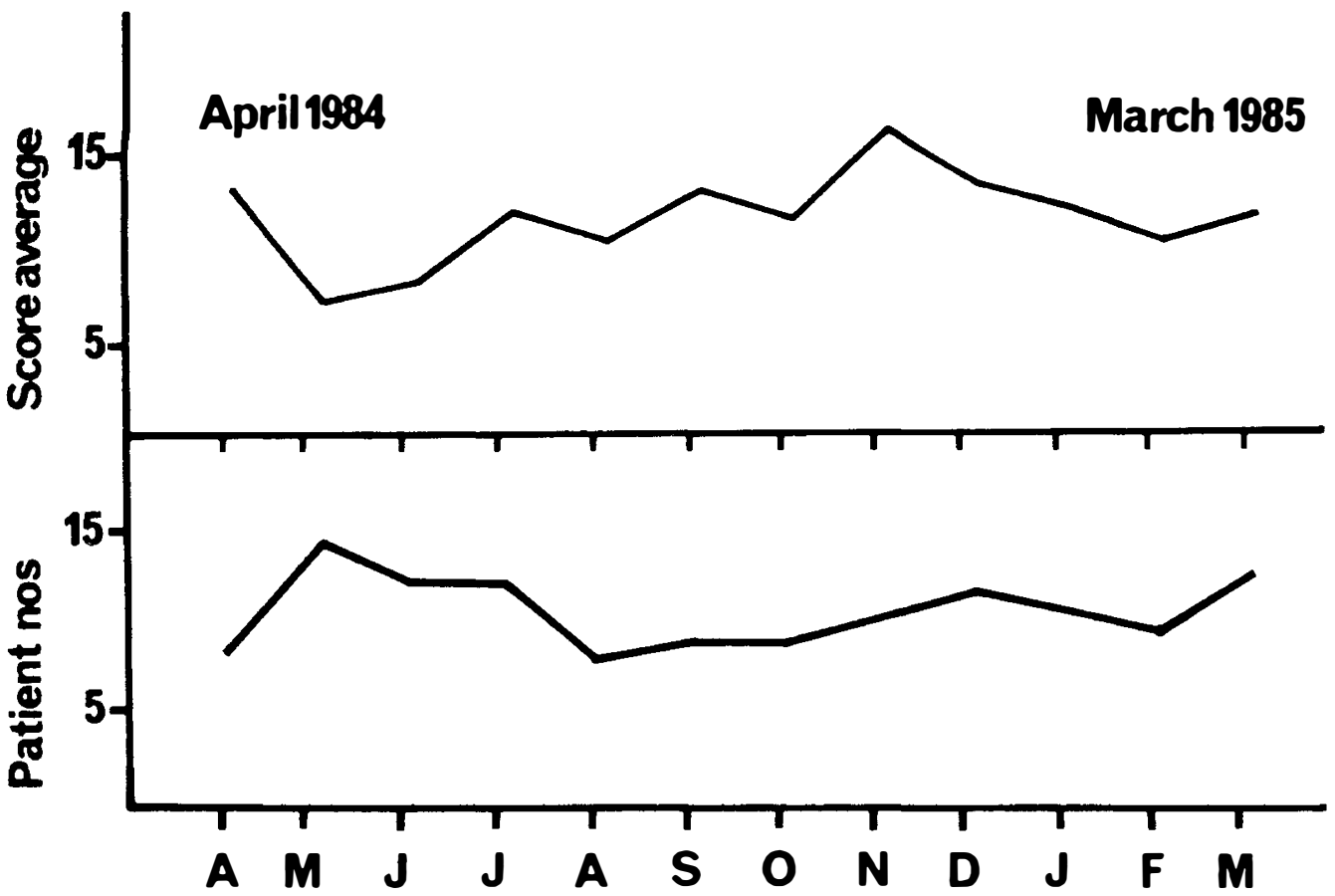

Fig. 5. Zoster clinic attendances and average clinical 'score' per month over the same year period.

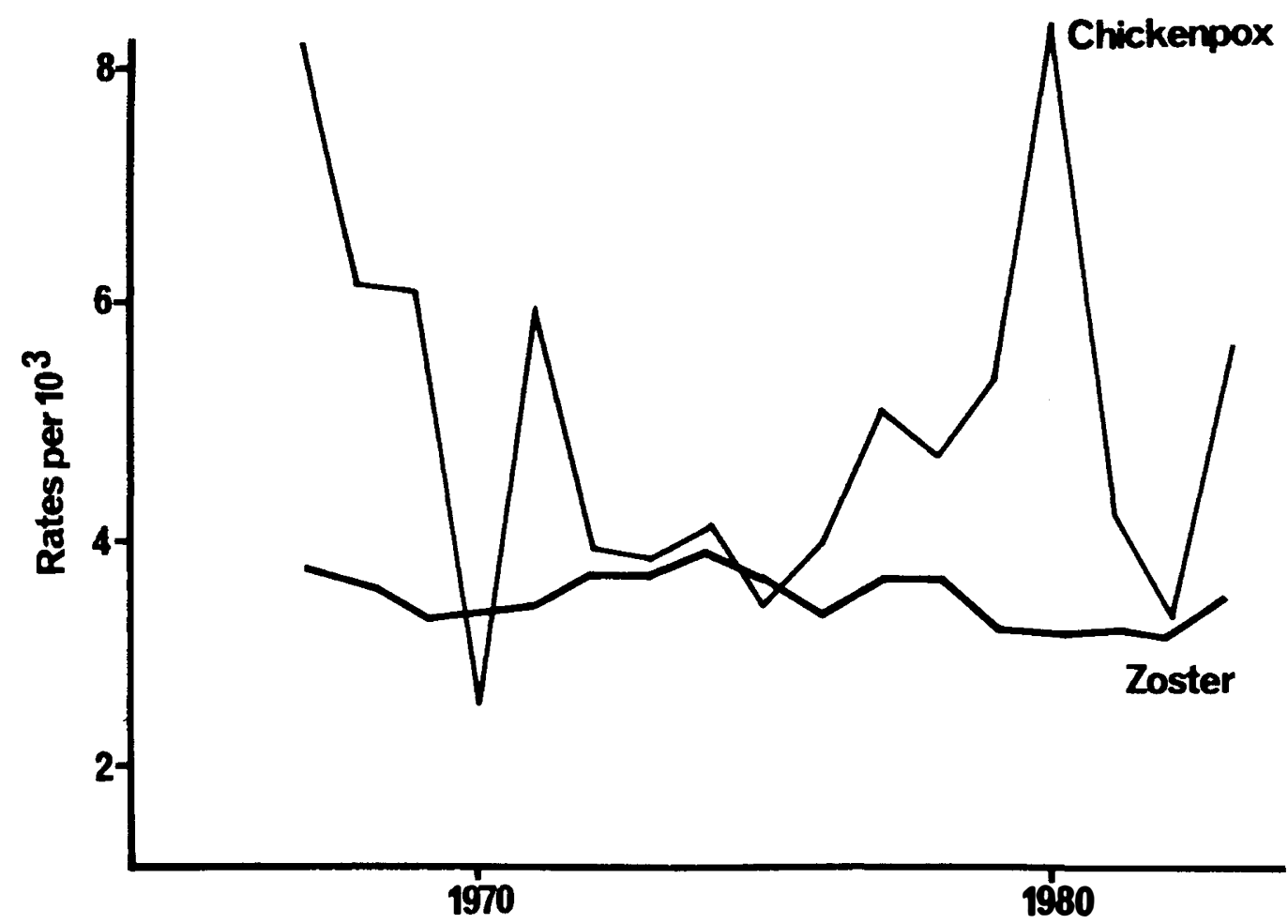

Fig. 6. Incidence rates for chickenpox and zoster per year 1967-1983. 


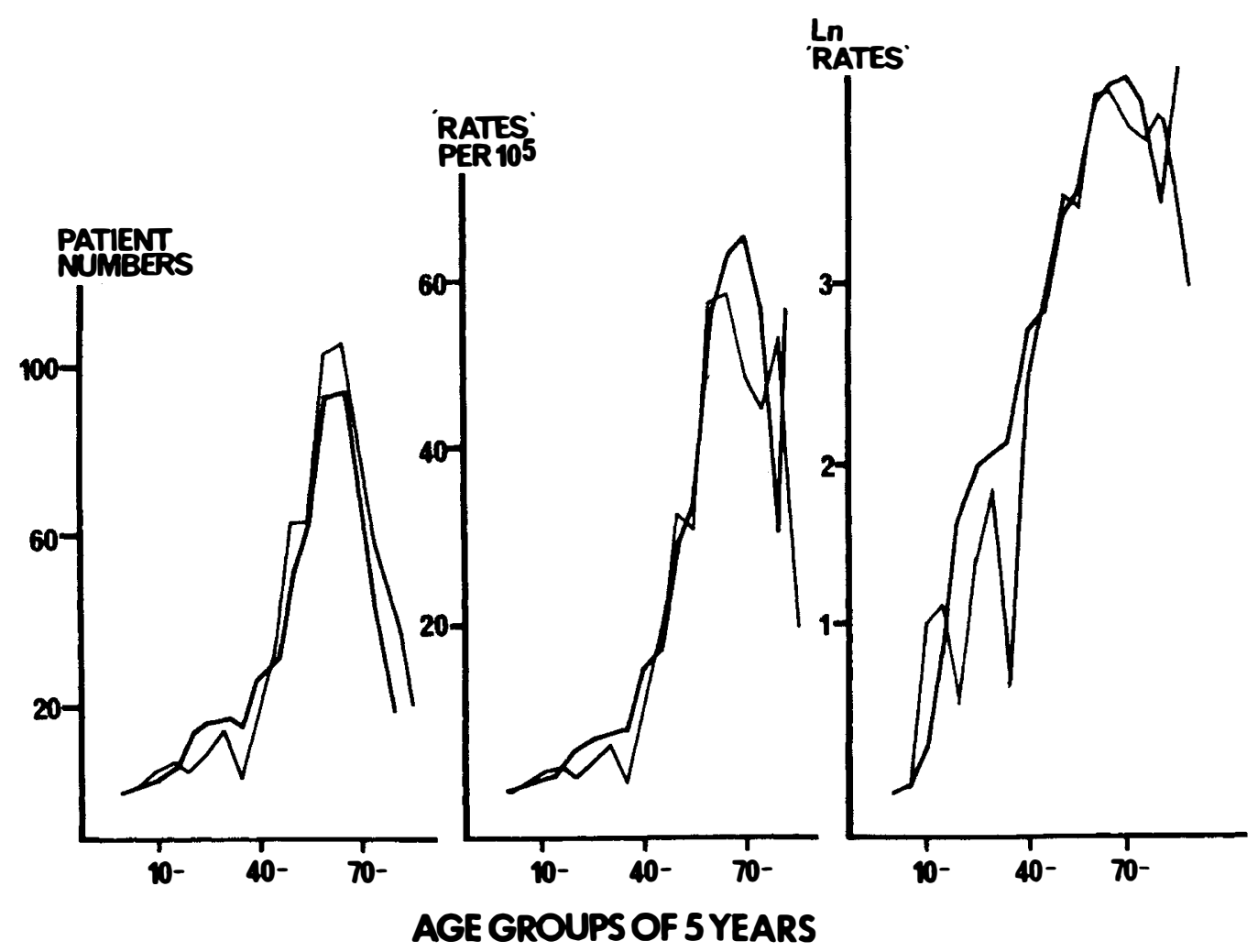

Fig. 7. Zoster clinic patients displayed by 5 year age groups. Males drawn with thick line, females with a thin one. Left: raw numbers. Middle: weighted for population structure. Right: ln vertical scale (ln = natural logarithm).

to threshold) increases exponentially as this level is decreased. In the Mortality model, 'energies' are represented by the various stresses to which people are subject and the threshold the ability with which the individual can deal with them: as this ability decreases with age, the number of stresses which cannot be dealt with successfully (and which lead to the death) will increase exponentially.

The plots shown are not linear (as would be predicted by the mortality models) but have a similar shape to cumulative normal distribution curves: that for cancer fits the proposed shape very accurately. The centre of this curve is at the 45-49 year age group and this is why the other curves were moved to correspond at this point, their different shapes being explained by alteration of basic parameters which govern particular disease and population characteristics. What these curves might be describing is the pattern of threshold decrease with age: the young have a high threshold and are only susceptible to the very occasional severe stress but the elderly have much lower levels and are affected by many weaker stresses. For many diseases the threshold reaches a plateau. Others have only just begun to do so by 80 years and these seem to be the main causes of death: they may represent common stresses or particularly low thresholds.

The $\mathrm{HZ}$ curve follows a similar pattern at least in the over-45 groups, so in $\mathrm{HZ}$ stresses could have the same effect as for mortality and the threshold govern the appearance of disease (rather than death). This model differs from previous ones in that another factor, loosely termed stress, is introduced to compound the age-dependence of threshold (however that might be represented). Going back to the clustering of $\mathrm{HZ}$ cases, ${ }^{1}$ it is quite possible to have clustering of 'stresses' (including a high exposure to VZV) which will have the same end result as viral infection. An analogy may be used to illustrate this model: if the state of a person's resistance to Zoster is 


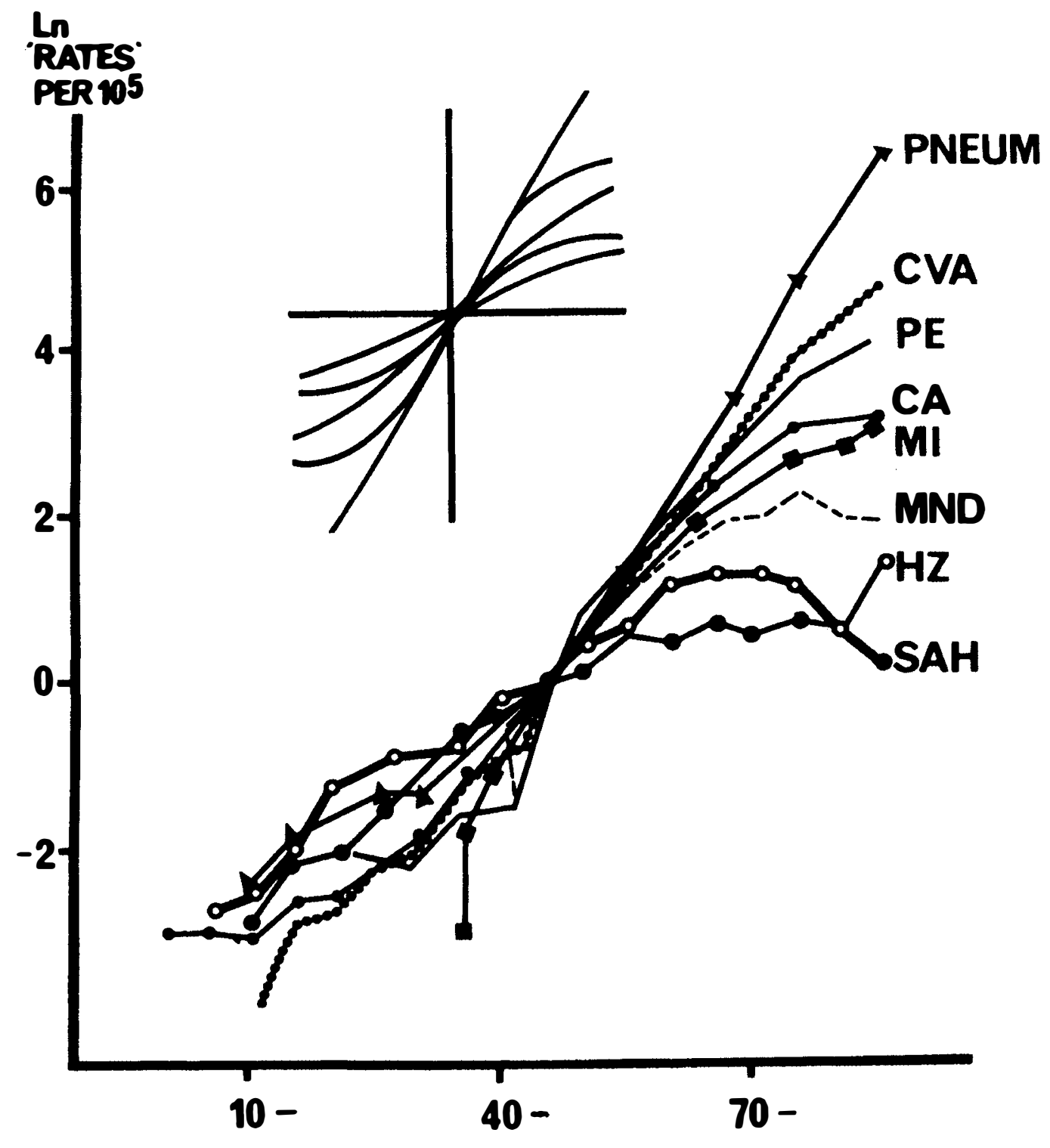

\section{AGE GROUPS OF 5 YEARS}

Fig. 8. Male zoster curve displayed with mortality curves, per 5 year age groups, for Pneumonia, CerebroVascular Accident, Pulmonary Embolus, Carcinoma, Myocardial Infarct, Motor Neurone Disease, and SubArachnoid Haemorrhage, shifted to correspond at the 45-49 year age group. Inset shows a family of cumulative Normal Distributions. 
represented by a cliff-top path and the stresses by a blustery cross-wind blowing out to sea, with increasing age the path nears the edge (in proportion to the height of the cumulative normal curve) so that the chance of a strong gust tipping them over the edge increases. The strength of the gusts will follow the exponential function.

It is interesting to see how non-specific factors could have a deciding role on whether a specific disease is expressed or not, and how general is the behaviour. Despite producing a degree of intellectual satisfaction, it is only a model and the data may well support other, perhaps more suitable ones. What is perhaps more challenging is what underlies these rather nebulous concepts and their implications for treatment or prevention.

I am grateful to Mr. R. Marsh for collecting the majority of the data over the last 15 years and supervising the evolution of this paper, to the RCGP for their figures and prompt assistance, to Mr. C. Kirkness for guidance and to Stephanie of the computing facility at Moorfields for her help.

\section{References}

${ }^{1}$ Palmer SR, Caul EO, Donald DE et al.: An outbreak of shingles: Lancet 1985; ii: 1108-10.
2 Peto TEA, Gilks CF, Juel-Jensen BE: Letter. Lancet 1985; ii: 1433

${ }^{3}$ Pallet A, Nicholls NWN: Varicella-Zoster: Reactivation or infection? Lancet 1986; i: 160.

${ }^{4}$ Palmer SR, Tillet H: Letter Lancet 1986; i: 273.

${ }^{5}$ Hope-Simpson RE: The nature of Herpes Zoster: A long term study and a new hypothesis. Proc. Roy. Soc. Med. 1965; 58: 9-20.

${ }^{6}$ Oxman MN: Varicella and Herpes Zoster. In: Dermatology in General Medicine Ed. Fitzpatrick et al. Mcgraw-Hill 1979.

7 Juel-Jensen BE, MacCullum FO: Herpes Simplex, Varicella and Zoster Philadelphia, Lippincott 1972.

${ }^{8}$ The Weekly Returns Service of the Royal College of General Practitioners: Chickenpox and Herpes Zoster 1982-1985.

- Office of Population Census and Surveys: Registrar General's Weekly Returns Service for England and Wales 1983-1984 London: HMSO.

${ }^{10}$ Office of Population Census and Surveys: Registrar General's Weekly Returns Service for England and Wales 1979-1980 London: HMSO.

${ }^{11}$ Fleming DM, Crombie DL: The Incidence of Common Infectious Diseases: The Weekly Returns Service of the RCGP. Health Trends 1985; 17: 13-16.

12 Office of Population Census and Surveys: 1981 Census HMSO.

${ }^{13}$ Office of Population Census and Surveys: 1981 Mortality Returns HMSO. 